\title{
The Comparison of Bone Scan and MRI in Osteoporotic Compression Fractures
}

\author{
Jung-Hoon Kim¹, Jong-In Kim¹, Bo-Hoon Jang ${ }^{1}$, Jung-Gook Seo², Jin-Hwan Kim¹ \\ 'Department of Orthopedic Surgery, Ilsan Paik Hospital, Inje University College of Medicine, Goyang, Korea \\ ${ }^{2}$ Department of Orthopedic Surgery, Seoul Paik Hospital, Inje University College of Medicine, Seoul, Korea
}

Study Design: Retrospective study.

Purpose: To estimate the usefulness of bone scan and magnetic resonance imaging (MRI) for the diagnosis of new fracture in osteoporotic vertebral fractures.

Overview of Literature: The diagnosis of new fractrure in osteoporotic vertebral fractures requires simple X-ray and supplementary studies.

Methods: We analyzed 87 vertebrae in 44 patients, who diagnosed with osteoporotic vertebral fractures using bone scan and MRI within 2 months interval between August 2001 and July 2008. We compared hot uptakes in bone scan with MRI findings such as new fractures, old fractures and degenerative lesions.

Results: Hot uptakes in bone scan was matched to 48 new fractures, 26 old fractures and 13 degenerative lesions in MRI findings. It was $55 \%$ of concordance between hot uptakes in bone scan and new fractures in MRI. The rate of new vertebral fractures confirmed by MRI according to 1 level hot uptakes in bone scan was 96\%, 2 levels was 50\% and 3 more levels was $36 \%$.

Conclusions: The diagnosis of new fracture in osteoporotic vertebral fractures requires simple X-ray and supplementary studies such as bone scan and MRI. We recommend more careful interpretation in multiple osteoporotic vertebral fracture patients about hot uptake lesions of bone scan.

Key Words: Osteoporotic vertebral fractures, Bone scan, MRI

\section{Introduction}

Vertebral compression fracture due to osteoporosis is a common cause of back pain in the elderly. The incidence and risk of vertebral compression fracture have recently shown a trend to rise due to the increased number of elderly people. For such osteoporotic vertebral compression fracture patients, it is prerequisite to accurately diagnose the symptomatic vertebra prior to deciding the treatment proto- cols. However, it is not easy to find the symptomatic vertebra with using only simple X-rays in the patients whose time of fracture onset and site of pain are difficult to determine, and in the elderly patients for whom it is difficult to perform a physical examination. Simple X-rays as well as computed tomography (CT) are primarily performed in elderly patients with osteoporotic vertebral compression fracture and for whom the trauma history is difficult to assess, yet supplement tests such as a bone scan and magnetic resonance imaging (MRI) are sometimes performed to

Received Dec 19, 2009; 1st Revised Jan 24, 2010; 2nd Revised Feb 9, 2010; Accepted Feb 9, 2010

Corresponding author: Jin-Hwan Kim, MD

Department of Orthopedic Surgery, Ilsan Paik Hospital, Inje University College of Medicine,

2240 Daehwa-dong, IlsanSeo-gu, Goyang 411-706, Korea

Tel: +82-31-910-7968, Fax: +82-31-910-7967, E-mail: jhkim@paik.ac.kr

* This work was supported by grant from Inje University, 2006. 
determine the symptomatic vertebra. There are scant reports that have compared the usefulness of several supplement tests for elderly patients without an apparent trauma history and who are suspected to have osteoporotic vertebral compression fracture. Maynard et al. [1] have reported that studies are needed to compare MRI with bone scans for detecting osteoporotic vertebral compression fracture. Therefore, we evaluated the usefulness of bone scanning and MRI to diagnose a new fracture, which is the symptomatic vertebra, in elderly patients with osteoporotic vertebral compression fracture.

\section{Materials and Methods}

Among the patients diagnosed with osteoporotic vertebral compression fracture from August 2001 to July 2008, 44 patients ( 87 vertebral bodies) underwent a bone scan and MRI within a 2 month period, and we retrospectively examined and analyzed these patients. The mean age of the patients was 72 years (range, 51 to 85 years), and there were 5 male patients and 39 female patients. The mean bone mineral densitometry was - 3.4 (range, - 1.3 to - 5.5). Bone scanning was performed on an average of 23 days (range, 6 to 83 days) from the day of manifesting symptoms, and MRI was performed on an average of 30 days (range, 2 to 99 days) from the day of manifesting symptoms. Simple X-rays were obtained at the time of admission. For the area with tenderness on the physical examination, the presence of vertebral body compression fracture was assessed by simple X-rays, and a bone scan was then performed to confirm it. MRI was additionally performed for the cases that required differentiation of simple compression fracture from other metabolic diseases, or for the cases that required the examination of other adjacent tissues.

According to the classification of Yamato et al. [2], new fracture and old fracture on MRI were distinguished by the change of signal intensity as represented by a geographic
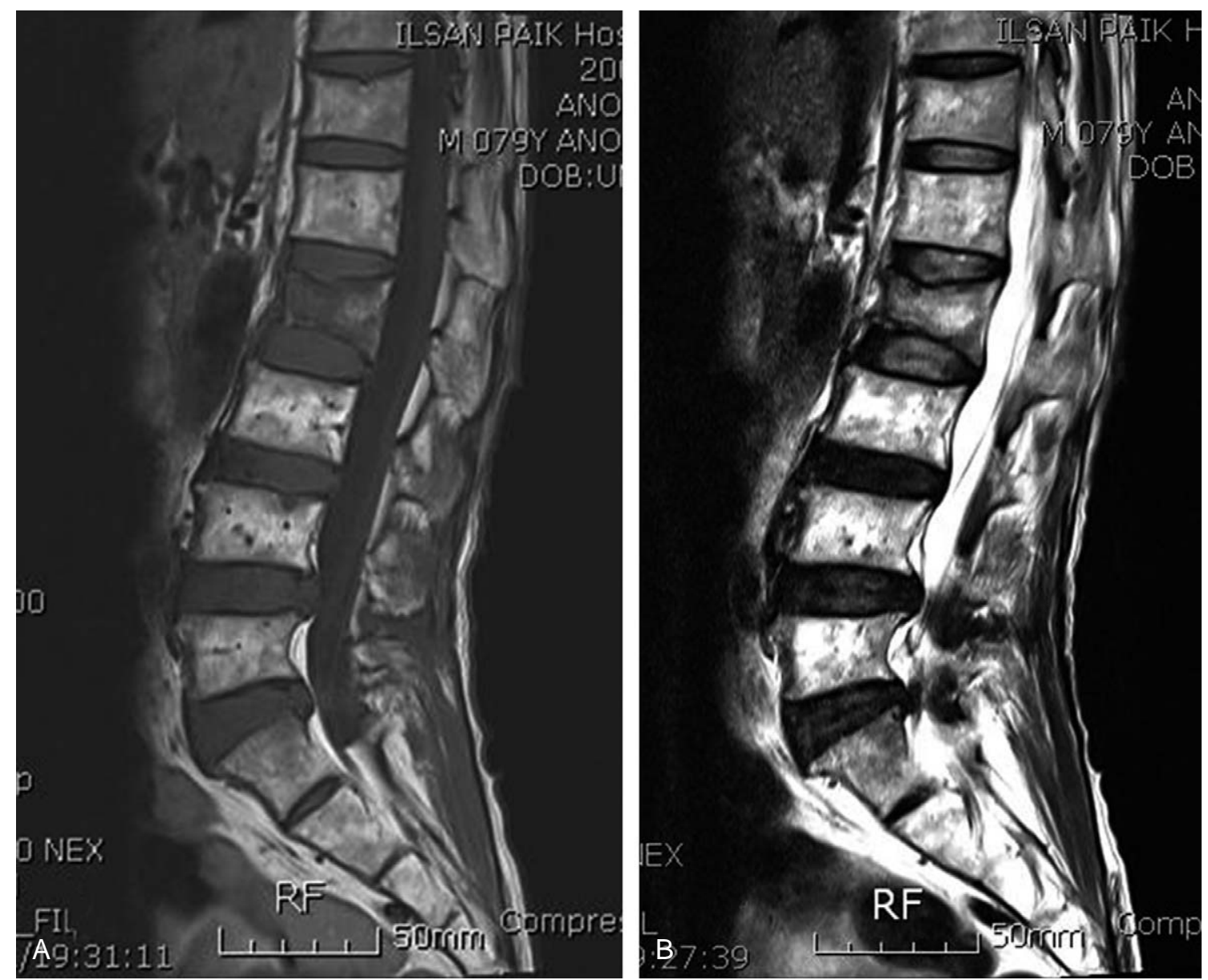

Fig. 1. Sagittal T1 weighted image (A) shows geographic areas of low intensity in L2 vertebral body. Sagittal T2 weighted image (B) shows geographic areas of high intensity. 
pattern or a linear pattern. The cases showing a geographic pattern that exhibited low intensity on the T1-weighted image and high intensity on the T2-weighted image were new fracture (Fig. 1). The cases showing low intensity on the T1-weighted image and this changed to high intensity or low intensity on the T2-weighted image as a linear pattern, and particularly normal bone marrow intensity was seen on the T1-weighted image, were determined to be old fracture (Fig. 2). In addition, the cases showing signal intensity limited to the vicinity of the upper and lower end plates of a vertebral body were determined to be disc degenerative lesions (Fig. 3).

The cases whose intensity on the bone scan was increased more than the hot uptake by the anterior superior iliac spine and the posterior superior iliac spine were diagnosed as hot uptake lesions (Fig. 4). Depending on the number of the vertebral bodies showing hot uptake lesions, the cases were divided into a single vertebral body group, a 2 vertebral body group and a more than 3 vertebral body group. All the lesions were compared and evaluated depending on the change of signal intensity on MRI, as well as the shape and the signal intensity of the new fractures, old fractures and degenerative lesions.

For the statistical analysis, chi-square tests were performed using the MedCalc ver. 11.1.1 (MedCalc Software, Mariakerke, Belgium), and a $p$-value less than 0.05 was considered to be significant.

\section{Results}

Among the 44 patients ( 87 vertebral bodies) who underwent a bone scan and MRI, 29 patients (48 vertebral bodies) showed a hot uptake by the bone scan and acute fracture by the MRI. Fifteen patients (39 vertebral bodies) showed a hot uptake by the bone scan, but no new fracture by MRI, and only $55 \%$ of the hot uptake lesions determined by a bone scan were confirmed to be new fracture by MRI.

Among the 24 patients ( 24 vertebral bodies) who were determined by bone scanning to have a hot uptake lesion in a single vertebral body, 23 patients ( 23 vertebral bodies) were determined to have a new fracture by MRI. The discrepant 1 patient ( 1 vertebral body) was found to have a
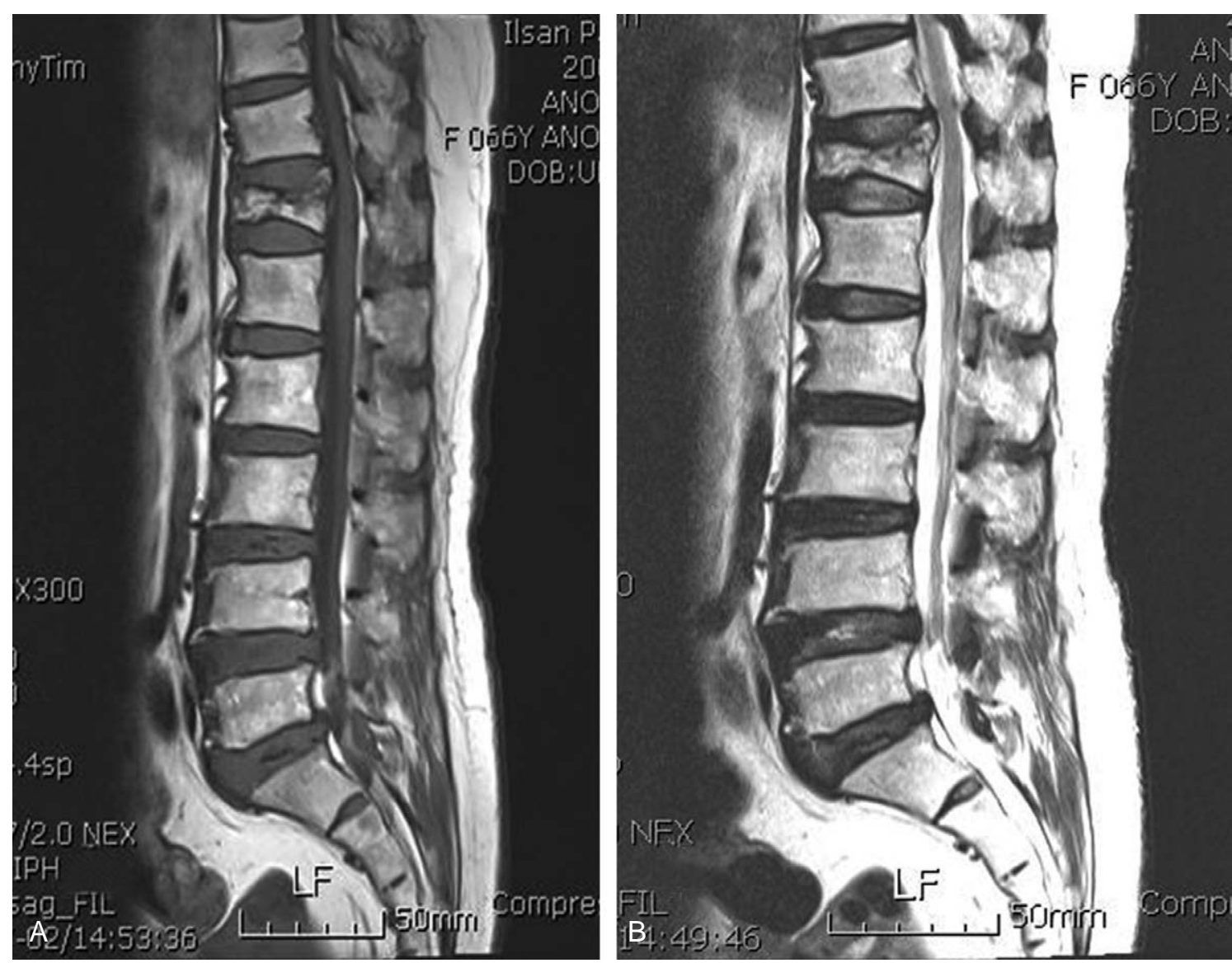

Fig. 2. Sagittal T1 weighted (A) and T2 weight (B) images show linear areas of low intensity with bone marrow replacement in T12 vertebral body. 
degenerative lesion by MRI. Six patients ( 9 vertebral bodies) among the 9 patients (18 vertebral bodies) with hot uptake in 2 vertebral bodies showed new fracture by MRI. Among the 9 discrepant vertebral bodies, 4 vertebral bodies had old fracture and 5 vertebral bodies were observed to have degenerative lesions. Among the 45 vertebral bodies with hot uptake lesion in more than 3 vertebral bodies, the concurrent cases were 16 vertebral bodies. Among the 29 discrepant vertebral bodies, 22 vertebral bodies had old fracture and 7 vertebral bodies were found to have degenerative lesions (Table 1).

Among the 24 patients determined to have hot uptake in a single vertebral body by a bone scan, 23 patients were determined to have new fracture by MRI. Among the 9
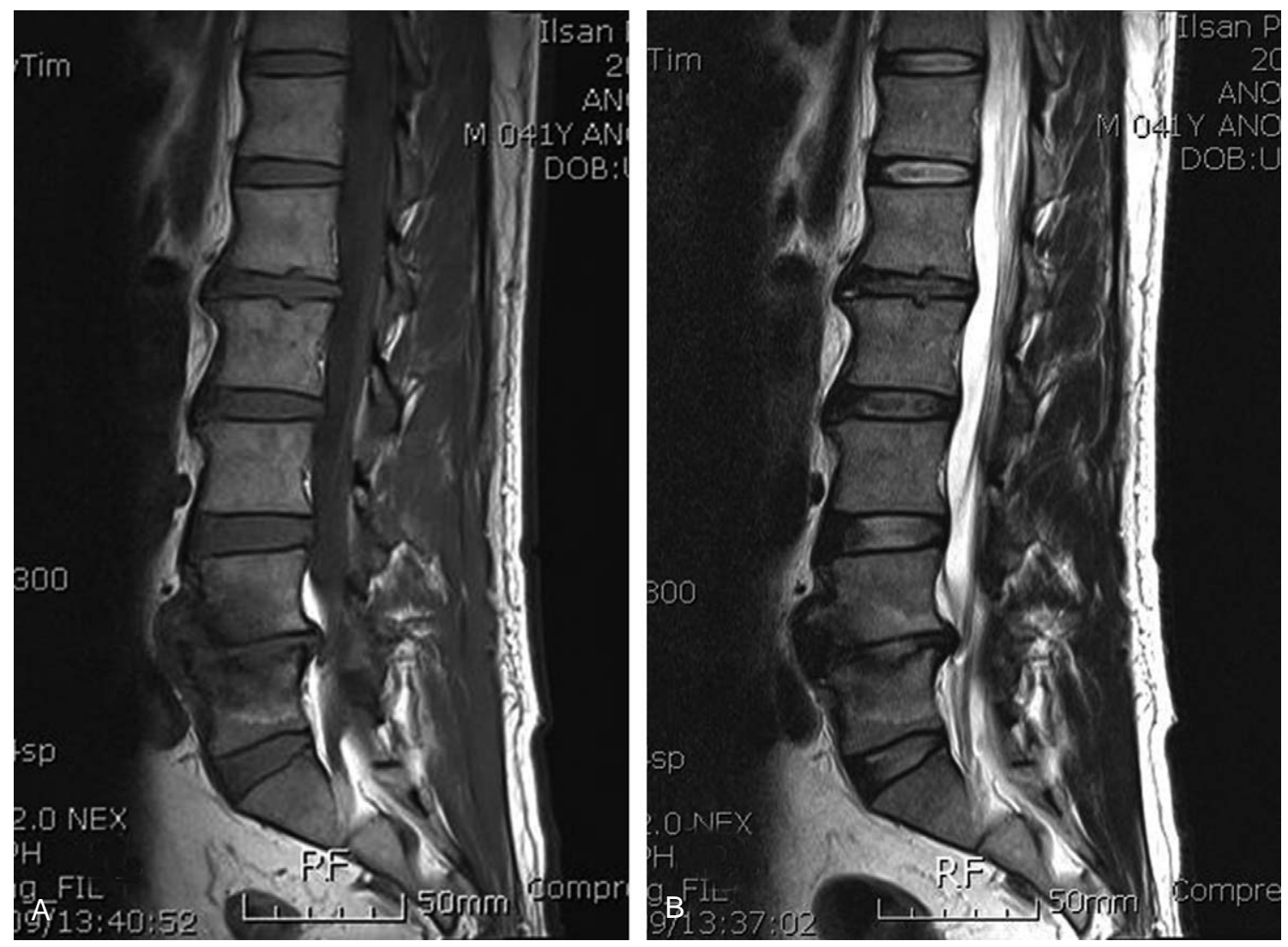

Fig. 3. Sagittal T1 (A) weighted image shows low intensity change above and below L4-5 disc and T2 (B) weighted image shows high intensity change.

Table 1. Magnetic resonance imaging (MRI) findings of the vertebrae based on the number of hot uptakes in bone scan

\begin{tabular}{lccc}
\hline \hline MRI findings & 1 level $(\mathrm{n}=24)$ & 2 level $(\mathrm{n}=18)$ & 3 more level $(\mathrm{n}=45)$ \\
\hline Recent fractures & 23 & 9 & 16 \\
Old fractures & 0 & 4 & 22 \\
Degenerative changes & 1 & 5 & 7 \\
\hline
\end{tabular}

n: Number of vertebra.

Table 2. The number of patients with recent vertebral fractures confirmed by magnetic resonance imaging (MRI) according to the number of hot uptakes in bone scan

\begin{tabular}{lccc}
\hline \hline MRI findings/Bone scan & 1 level $(\mathrm{n}=24)$ & 2 level $(\mathrm{n}=9)$ & 3 more level $(\mathrm{n}=11)$ \\
\hline 1 level recent fractures & 23 & 3 & 6 \\
2 level recent fractures & & 3 & 2 \\
3 level recent fractures & & & 2 \\
\hline
\end{tabular}

$\mathrm{n}$ : Number of patient. 
patients shown to have hot uptake in 2 vertebral bodies, 3 patients showed new fracture findings in a single vertebral body by MRI, and 3 patients showed new fracture in 2 vertebral bodies. Among the 11 patients who showed hot uptake in more than 3 vertebral bodies, 6 patients were shown to have new fracture in a single vertebral body by MRI (Fig. 5), 2 patients were shown to have new fracture in 2 vertebra bodies by MRI and 2 patients were shown to have new fracture in 3 vertebral bodies by MRI (Table 2). Ninety six percent, $50 \%$ and $36 \%$ of the cases with a hot uptake on a bone scan in 1,2 and 3 more than vertebral bodies, respectively, were confirmed to be new fracture by MRI. We found that when hot uptake lesions were detected in more than 2 vertebral body, the possibility of confirming this as new fracture via MRI became lower, and this was statistically significant $(p<0.001)$ (Table 3$)$.

\section{Discussion}

Simple X-ray and CT have been important diagnostic tests for making the initial diagnosis of osteoporotic vertebral compression fracture. CT could be a convenient diagnostic test for determining the presence or absence of frac- ture. Nonetheless, making the differential diagnosis of old fracture and new fracture can not be clearly done in many cases. Hence, bone scan and MRI are often performed for the elderly patients with vertebral disease and who are with-

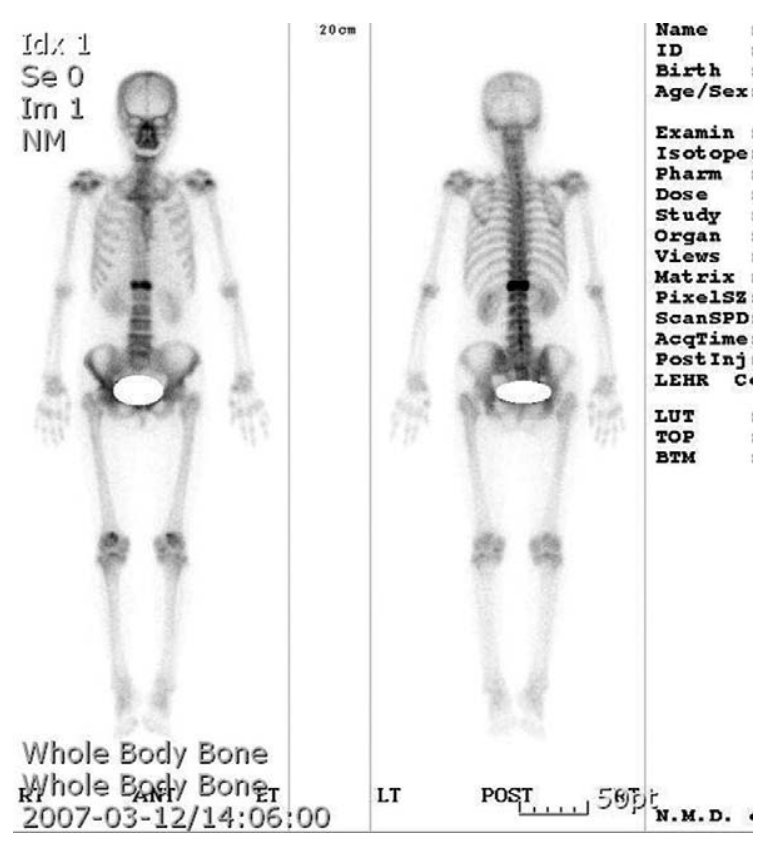

Fig. 4. T12 vertebra hot uptake in bone scan.
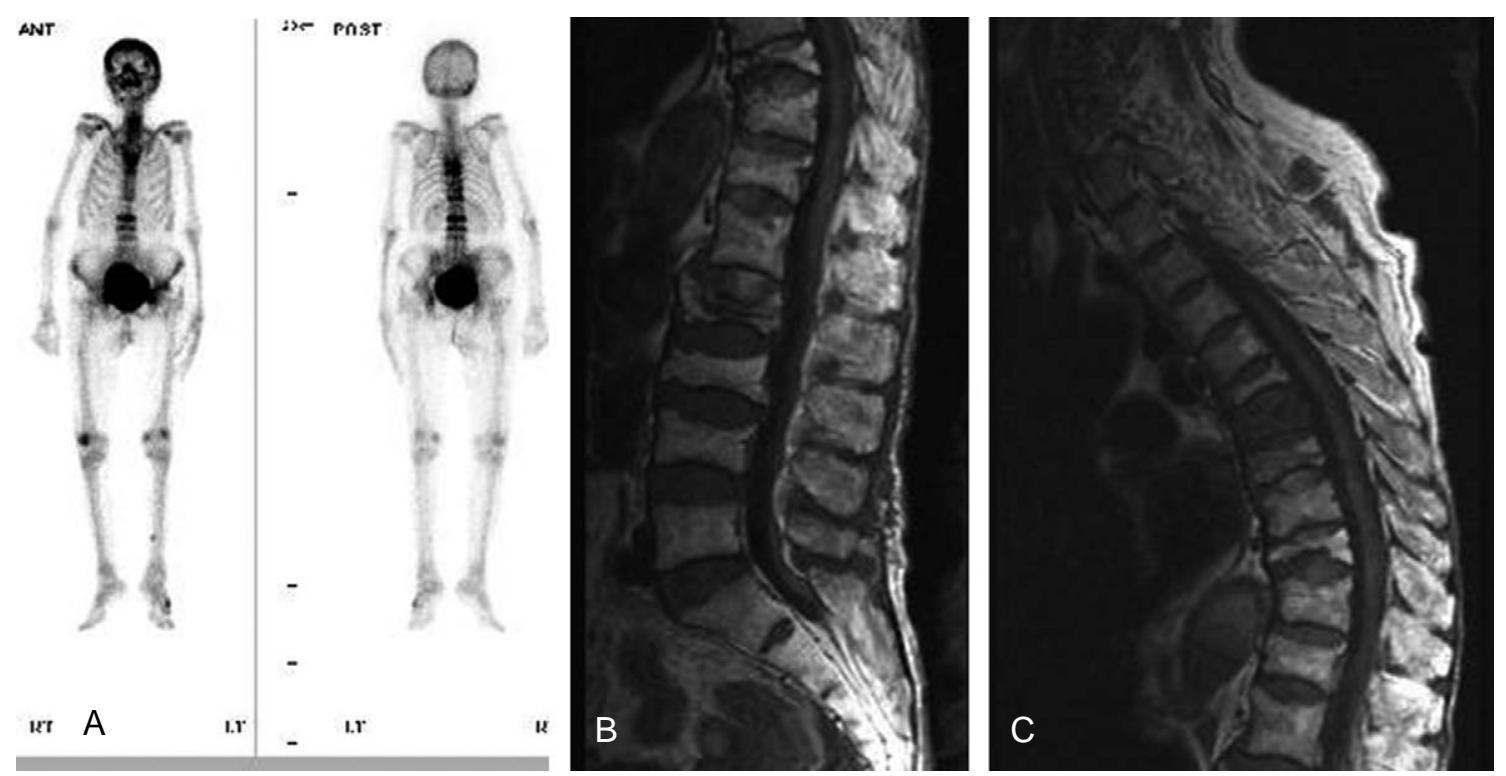

Fig. 5. Bone scan (A) hot uptakes in T7, 8, 9, 10, 11, 12, L2, 3 vertebra, thoracic magnetic resonance imaging sagittal T1 weighted (B) and lumbar (C) images show geographic areas of low intensity in only T7 vertebral body.

Table 3. The rate of vertebral fractures confirmed by MRI according to the number of hot uptakes in bone scan

\begin{tabular}{ccccc}
\hline \hline MRI findings/Bone scan & 1 level $(\mathrm{n}=24)$ & 2 level $(\mathrm{n}=18)$ & 3 more level $(\mathrm{n}=45)$ & $p$-value \\
\hline Recent fractures & $96 \%$ & $50 \%$ & $36 \%$ & 0.001 \\
\hline
\end{tabular}

MRI: Magnetic resonance imaging, n: number of vertebra. 
out a definite trauma history.

Since a bone scan reflects the bone metabolism, it may play an important role in the differentiation of old fractures and to detect metabolic diseases or fracture that is undetected by simple X-rays. Matin [3] has reported that for the cases of vertebral body fracture found on a bone scan, $90 \%$ showed normal findings within 2 years and $97 \%$ showed normal findings within 3 years. According to Kim et al. [4], from day 10 after spinal and pelvic bone fractures, the probability of seeing a positive reaction on a bone scan is high, and despite that there is an apparent fracture, the fracture is shown as negative on a bone scan in some cases. Thus, fracture could not be completely ruled out even if the findings are negative on a bone scan [4]. Maynard et al. [1] have reported that bone scanning better represented the function of bone metabolism and it could shorten the test time, and so it is preferred to MRI. Yet the hot uptake on a bone scan persists for 2 years after fracture, and so a bone scan was not of great help for diagnosing vertebral body fracture older than 6 months [1]. Cook et al. [5] have reported that for osteoporosis patients who present with back pain, the bone scan could distinguish the cause of back pain among fracture, facet joint arthritis and disc degenerative lesions and so it is of great help to administer appropriate treatments, but Cook et al. [5] didn't suggest clear objective standards that distinguish individual diseases.

Images in several planes can be obtained by MRI, and MRI can be an important diagnostic test to assess soft tissue injuries. In addition, depending on the presence or absence of the change of signal intensity and the altered patterns, accurate delineation of pathological areas can be confirmed by MRI. Furthermore, new fracture, old fracture and degenerative lesions can be differentiated because of MRI's good resolution. According to the study reported by Baker et al. [6], due to the swelling or inflammation, acute traumatic compression fracture shows low intensity on T1-weighted images and high intensity on T2-weighted images, and upon entering the chronic phase at 1-3 months after injury, the intensity of the vertebral body was normalized. Frager et al. [7] also reported that for acute and subacute osteoporotic vertebral compression fracture, low intensity was observed on the T1-weighted images and high intensity was observed on the T2-weighted images. Acute fracture causes bleeding and edema, and it increases the local water content, and so high intensity is seen on the T2-weighted images. When the bleeding becomes organized and the edema subsides, low intensity or isosignal intensity is observed on the T2- weighted images [7]. Do [8] has reported that the selection of appropriate patients is important for the success of surgery, and preoperative MRI is useful for this, and according to the change of signal intensity, the lesion could be classified to acute, subacute and chronic fracture, and pathologic fractures such as metastatic lesions could be differentiated. In addition, Yamato et al. [2] established a quantitative standard for the ratio of the change of signal intensity in the vertebral body by classifying the change of signal intensity into geographical patterns as G1 (change in the entire vertebral body), G2 (change in more than $50 \%$ of the vertebral body), G3 (change in $25-50 \%$ of the vertebral body), and G4 (change in less than $25 \%$ of the vertebral body), and such change of signal intensity as geographical patterns gradually lessened after the acute phase, and linear changes were substituted after 2-5 months.

In our study, $55 \%$ of the cases that showed hot uptake on a bone scan were confirmed to have new fracture by MRI, and only in approximately more than half cases was the lesion observed on a bone scan found to concur with a lesion observed on MRI. In addition, for the cases showing hot uptake in a single vertebral body on a bone scan, the possibility of this being a new fracture as assessed on MRI was $96 \%$, yet the concurrence rate was $50 \%$ for hot uptake in 2 vertebral bodies and $36 \%$ for hot uptake in more than 3 vertebral bodies. Thus, it was observed as the number of the vertebral bodies showing hot uptake lesions was increased, the possibility of observing new fracture on MRI was decreased. In cases with suspected solitary vertebral compression fracture, a bone scan could be usefully applied to detect a new fracture that is causing symptoms. Nonetheless, it is thought that in patients with compression fracture in more than 2 vertebral bodies, more attention has to be paid to accurately assess the fractured areas. In addition, it was found that for patients with compression fracture in more than 3 vertebral bodies, $49 \%$ of the hot uptake on the bone scan was old fracture, and so old fracture should be considered in the patients with fracture in more than 3 vertebral bodies, as observed on a bone scan.

The limitations of our study are that that quantitative analysis of the hot uptake lesion detected by bone scanning could not be performed and so we didn't obtain objective indexes by comparing the shapes and the changes of signal intensity detected on MRI. Further, the outcome of the administered treatments according to the result of each diagnosis could not be compared. Nevertheless, if diagnostic standards could be provided through prospective studies 
in the future, it could be of great help not only for assessing vertebral fracture, but also for making the differential diagnosis of other diseases. With such data, bone scanning could play an important role as an initial diagnostic tool and help decide the appropriate treatment protocols.

\section{Conclusions}

For solitary osteoporotic vertebral compression fracture, MRI and a bone scan may be useful as adjuvant diagnostic tools, in addition to simple X-rays, to find the vertebral body manifesting the symptoms. Nonetheless, for the fracture involving more than 2 vertebral bodies, the possibility of confirming the hot uptake lesion as a new fracture by bone scanning is low, and so MRI is required to make the diagnosis.

\section{REFERENCES}

1. Maynard AS, Jensen ME, Schweickert PA, Marx WF, Short JG, Kallmes DF. Value of bone scan imaging in predicting pain relief from percutaneous vertebroplasty in osteoporotic vertebral fractures. AJNR Am J Neuroradiol 2000;21:1807-12.

2. Yamato M, Nishimura G, Kuramochi E, Saiki N, Fujioka
M. MR appearance at different ages of osteoporotic compression fractures of the vertebrae. Radiat Med 1998; 16:329-34.

3. Matin P. The appearance of bone scans following fractures, including immediate and long-term studies. J Nucl Med 1979;20:1227-31.

4. Kim JY, Choi YA, Noh BK, Kong BS. The clinical significance of bone scan in fracture diagnosis. J Korean Soc Fract 1988;1:102-8.

5. Cook GJ, Hannaford E, See M, Clarke SE, Fogelman I. The value of bone scintigraphy in the evaluation of osteoporotic patients with back pain. Scand J Rheumatol 2002; 31:245-8.

6. Baker LL, Goodman SB, Perkash I, Lane B, Enzmann DR. Benign versus pathologic compression fractures of vertebral bodies: assessment with conventional spin-echo, chemical-shift, and STIR MR imaging. Radiology 1990; 174:495-502.

7. Frager D, Elkin C, Swerdlow M, Bloch S. Subacute osteoporotic compression fracture: misleading magnetic resonance appearance. Skeletal Radiol 1988;17:123-6.

8. Do HM. Magnetic resonance imaging in the evaluation of patients for percutaneous vertebroplasty. Top Magn Reson Imaging 2000;11:235-44. 